\title{
Taking aim at mosquitoes
}

\section{The malaria vector is back in scientists' sights, says Janet Hemingway, with insecticides and transgenic insects offering fresh hope.}

$\mathbf{N}_{\mathrm{i}}$ mosquitoes - no malaria. The insects are essential for transmitting the Plasmodium parasite, so for a century the mainstay of control was indoor residual spraying of insecticides. In the past decade, that has changed, and we have come to rely on antimalarial drugs as the main means of control. But with parasite resistance to cheap antimalarials growing, mosquito control is back on the agenda - and it will need new tools if it is to become a sustainable option.

Indoor spraying or bednets impregnated with pyrethroid insecticide can dramatically reduce death and illness, but a question mark hangs over their long-term effectiveness. Mosquito resistance to pyrethroids may hamper the use of impregnated bednets ${ }^{1}$. Our understanding of the effect of control on the interaction between malaria transmission rates, acquired immunity and disease incidence is poor. Children exposed to infected mosquito bites can build up immunity and maintain it as adults if regularly exposed. But if transmission drops, they can lose immunity and risk developing severe malaria if it increases again.

So control methods that cut mortality in the short term could make populations more vulnerable in the long term. Encouragingly, a recent study in Kenya showed that the benefits of bednets remained six years after their introduction ${ }^{2}$.

To make progress, existing methods will have to be deployed more effectively. In many countries malaria occurs mostly in the poorest, rural sectors of society, and even relatively simple control methods are rarely applied effectively. Fewer people competent in large-scale mosquito control are now working in Africa than 50 years ago, owing to the phasing out of national control programmes after malaria eradication campaigns failed in the 1950s and 1960s. To make

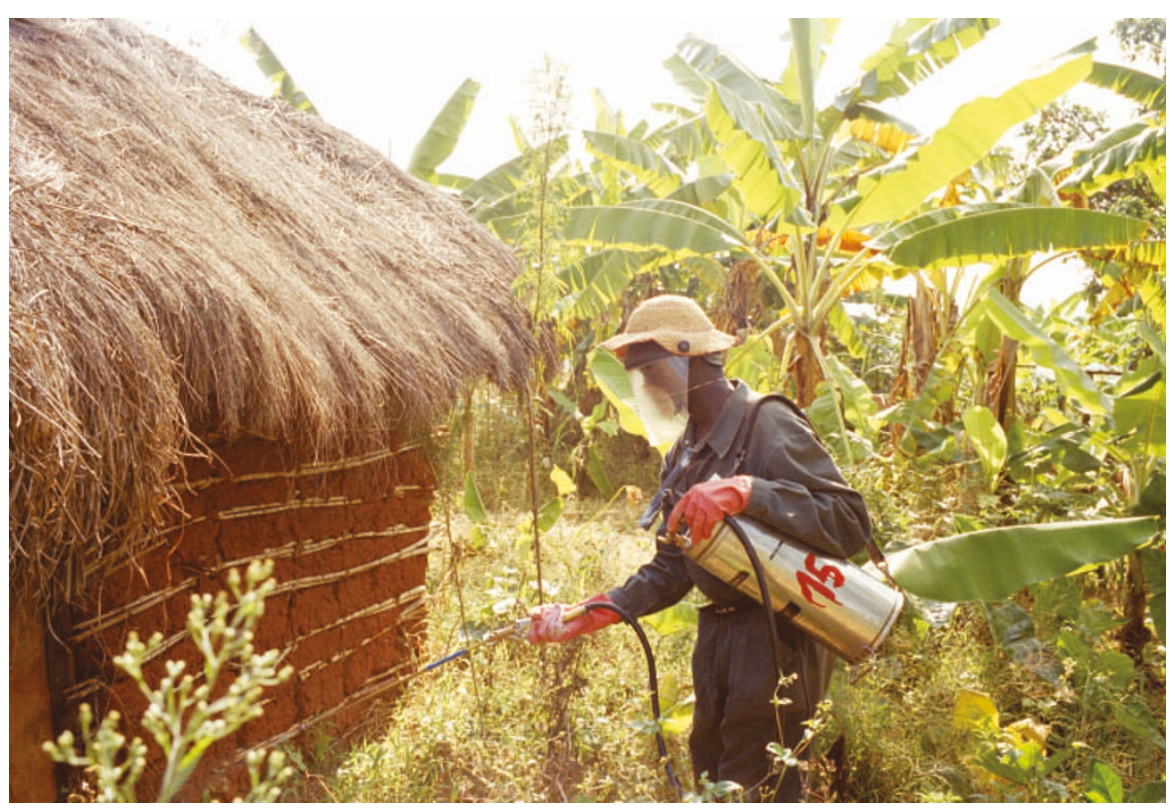

Making a splash: mosquito control plays an important part in reducing death and illness.

matters worse, insecticide resistance outpaces the appearance of new insecticides.

There are obvious ways to improve the way we tackle mosquitoes. Technologies such as remote sensing and climate modelling can map and monitor mosquito populations without the the need for armies of insect collectors. Electronic data collection and transfer could be better used to feed information to control managers in real time. Such tools are starting to improve understanding of malaria transmission dynamics, but they need to be adapted to provide useful data at local scales and made available to affected communities.

Another exciting possibility is to exploit the mosquito and parasite genome sequences $^{3,4}$ to develop insecticides that attack new targets within the vector or thwart resistance to existing pesticides. Such innovations could be in use within ten years.

\section{Mosquito modification \\ Other genetic approaches} include modifying mosquitoes to produce offspring that cannot transmit disease. Researchers have made great strides in this area. Introducing transgenics into the wild would not depend on health infrastructure. But where several species of vector are present, a separate transgenic must be created for each - a far from trivial undertaking. For example, it is still impossible to engineer Anopheles funestus, the major vector in southern Africa.

Alternatively we could use treatments based on RNAi (double-stranded RNA fragments that block the expression of specific genes) to 'silence' mosquito genes. Comparisons of the Drosophila and Anopheles gambiae genomes have identified several mosquito gene families, such as C-type lectins, that have undergone recent expan$\operatorname{sion}^{5,6}$ and are potential targets. Transcriptional analysis of Plasmodium and its vector during parasite development ${ }^{7,8}$ is pinpointing genes unique to mosquitoes, suggesting we could block mosquito-parasite interactions without affecting other insects. Progress is rapid, but ideal candidates have yet to be identified ${ }^{6}$.

Scaling up these technologies presents further challenges. Most at-risk populations have a poor understanding of malaria transmission, so acceptance of measures often depends more on how well biting insects are controlled than on potential reductions in malaria. Political and social acceptance of the release of parasite-resistant mosquitoes will likewise be needed.

Alternatively, there may be ways to exploit the finely tuned relationship that has evolved between parasite and vector. Simply altering the redox state of the insect's gut, through which the parasite must pass, may stop transmission? ${ }^{9}$.

Although these openings give cause for optimism, they are only the first small steps along the difficult road to implementation. But such challenges should not diminish our determination to combat malaria.

Janet Hemingway is director of the Liverpool School of Tropical Medicine, Liverpool, UK.

\footnotetext{
1. Brooke, B. D. et al. Bull. Entomol. Res. 91, 265-273 (2001).

2. Lindblade, K. A. et al. J. Am. Med. Assoc. 291, 2571-2580 (2004).

3. Holt, R. A. et al. Science 298, 129-149 (2002)

4. Gardner, M. J. et al. Nature 419, 498-511 (2002).

5. Ranson, H. et al. Science 298, 179-181 (2002).

6. Osta, M. A., Christophides, G. K. \& Kafatos, F. C. Science 303, 2030-2032 (2004).

Abraham, E. G. et al. J. Biol. Chem. 279, 5573-5580 (2004).

8. Srinivasan, P. et al. J. Biol. Chem. 279, 5581-5587 (2004).

9. McCarroll, L. et al. Nature 407, 961-962 (2000).
} 